\title{
Curcumin-induced promoter hypermethylation of the mammalian target of rapamycin gene in multiple myeloma cells
}

\author{
JIAQI CHEN $^{1}$, YONGLI YING ${ }^{2}$, HONGJUN ZHU ${ }^{1}$, TINGJUN ZHU ${ }^{3}$, \\ CHUNSHENG QU ${ }^{1}$, JINHONG JIANG ${ }^{3}$ and BINGMU FANG ${ }^{3}$ \\ ${ }^{1}$ Clinical Laboratory; Departments of ${ }^{2}$ Surgery and ${ }^{3}$ Hematology, Lishui People's Hospital, \\ Sixth Affiliated Hospital of Wenzhou Medical University, Lishui, Zhejiang 323000, P.R. China
}

Received January 18, 2018; Accepted October 3, 2018

DOI: $10.3892 / \mathrm{ol} .2018 .9662$

\begin{abstract}
Curcumin, a polyphenol derived from the rhizome of Curcuma, is a potential tumor inhibitor through affecting signaling pathways and epigenetic regulation. The mammalian target of rapamycin (mTOR) gene serves a crucial role in the carcinogenesis of multiple myeloma. The curcumin-induced epigenetic regulation of mTOR, including promoter DNA methylation in multiple myeloma, has not yet been fully elucidated. In the present study, antitumor effects of curcumin were investigated in RPMI-8226 and NCI-H929 cells using an MTT assay and flow cytometry. The expression of mTOR and DNA methyltransferase proteins were determined by western blot analysis, and the methylation status of the mTOR promoter were detected by sequencing following bisulfite conversion. The results of the present study revealed that the half-maximal inhibitory concentration of curcumin was $10 \mu \mathrm{M}$ in myeloma cells. Following curcumin treatment, the mRNA and protein expression levels of mTOR were decreased by 43.31 and $39.34 \%$ in NCI-H929 cells, respectively. The promoter of mTOR, located in chromosome 1 (chromosome position, 11262153-11263153), contains a $\mathrm{CpG}$ island that was hypermethylated in myeloma cells following curcumin treatment. The expression levels of DNA methyltransferase (DNMT)3a and DNMT3b were increased in curcumin-treated cells. Collectively, these results indicate that curcumin serves a role in the epigenetic regulation of mTOR expression, and that mTOR downregulation is due to promoter hypermethylation, which may be associated with DNMT3a and DNMT3b upregulation.
\end{abstract}

Correspondence to: Professor Bingmu Fang, Department of Hematology, Lishui People's Hospital, Sixth Affiliated Hospital of Wenzhou Medical University, 15 Dazhong Road, Lishui, Zhejiang 323000, P.R. China

E-mail: fbm636@163.com

Abbreviations: mTOR, mammalian target of rapamycin; DMNT, DNA methyltransferase; MECP2, methyl CpG-binding protein 2

Key words: curcumin, DNA methyltransferase 3, hypermethylation, mammalian target of rapamycin, myeloma cells
The results of the present study contribute towards improving the understanding of curcumin treatment in multiple myeloma and provide novel insights into the molecular mechanisms underlying the epigenetic regulation of mTOR.

\section{Introduction}

Multiple myeloma is a B-cell malignancy characterized by abnormal plasmacyte proliferation in the bone marrow (1). The morbidity contribution of multiple myeloma is $\sim 1 \%$ of all tumors and $10 \%$ of blood system tumors $(1,2)$. Multiple myeloma is the second most common malignancy of the blood system. An Australian study identified that the survival time for the majority of cases was between 5 and 8 years in elderly patients, accounting for $20 \%$ of hematological mortalities (2). Although various treatments have been tested, including thalidomide, lenalidomide, bortezomib and hematopoietic stem cell transplantation, multiple myeloma remains an incurable blood cell cancer $(3,4)$. Therefore, the development of more effective antitumor drugs and novel therapeutic strategies for multiple myeloma is urgently required.

Curcumin is a polyphenol derived from the rhizome of Curcuma, and has long been used for treating various types of cancer, including colorectal carcinoma, glioma and lung cancer, by inhibiting the proliferation and promoting the apoptosis of the cancer cells (5-9). As a potential cancer inhibitor, curcumin inhibits almost every phase of tumor development, including infiltration, colonization and outgrowth. Previous studies have demonstrated that the antitumor function of curcumin is associated with a range of genetic and epigenetic alterations of oncogenes and anti-oncogenes, including the RAC- $\alpha$ serine/threonine-protein kinase (AKT)/mammalian target of rapamycin (mTOR) pathway components and downstream signaling molecules $(10,11)$.

mTOR is known as the main regulatory factor of cell autophagy and apoptosis $(10,11)$, which are involved in the regulation of various cellular processes. Clinical studies have demonstrated that the inhibition of mTOR activation can promote cell apoptosis in several types of cancer, including pancreatic cancer, bladder cancer and leukemia (12-15). Furthermore, inhibition of the mTOR signaling pathway can enhance cytotoxicity and induce autophagy in a possible protective role in K562 leukemia cell lines and in chronic 
myeloid leukemia (16). Resveratrol, a natural phytoalexin, inhibits the mTOR pathway and induces cell apoptosis and autophagy in multiple myeloma (17).

Previous studies have suggested that curcumin, a constituent of Curcuma longa, is a potential epigenetic regulator. This polyphenol inhibits the activity of histone acetyltransferase, which can lead to inhibition of histone acetylation (18-20). Curcumin also blocks the catalytic C1226 site of DNA methyltransferase (DNMT)1 through its chemical structure to inhibit the activity of the enzyme (21). However, the curcumin-induced epigenetic regulation of biomarkers in multiple myeloma has not yet been fully understood, and specifically, whether mTOR serves a role in epigenetic regulation remains unknown. In the present study, curcumin was revealed to induce the methylation of the mTOR promoter, potentially via the upregulation of DNMT3a and DNMT3b.

\section{Materials and methods}

Cell culture and treatment. The NCI-H929 and RPMI-8226 cell lines were purchased from the American Type Culture Collection bio-resource center (Manassas, VA, USA). All cells were suspended, cultured and maintained in RPMI-1640 (Gibco; Thermo Fisher Scientific, Inc., Waltham, MA, USA) supplemented with $10 \%$ fetal bovine serum (FBS; Gibco; Thermo Fisher Scientific, Inc.), $100 \mathrm{U} / \mathrm{ml}$ penicillin and $100 \mu \mathrm{g} / \mathrm{ml}$ streptomycin (Gibco; Thermo Fisher Scientific, Inc.) at $37^{\circ} \mathrm{C}$ in a humidified $5 \% \mathrm{CO}_{2}$ atmosphere. Curcumin (Sigma-Aldrich; Merck KGaA, Darmstadt, Germany) was dissolved in dimethylsulfoxide (DMSO; Beyotime Institute of Biotechnology, Haimen, China) as stock solution (1 mg/ml).

For the proliferation assay, the cells were cultured in 96-well plates at $5 \times 10^{4}$ cells/well and treated with increasing concentrations $(0,5,10,15$ and $20 \mu \mathrm{M})$ of curcumin. At various time points $(0,24,48,72,96$ and $120 \mathrm{~h}), 10 \mu \mathrm{l}$ MTT solution (Beyotime Institute of Biotechnology, Haimen, China) was added to each well, then the cells were incubated for $4 \mathrm{~h}$ at $37^{\circ} \mathrm{C}$ in a humidified $5 \% \mathrm{CO}_{2}$ atmosphere. The formazan crystals that formed were dissolved with $100 \mu \mathrm{l}$ DMSO for $2 \mathrm{~h}$ at $37^{\circ} \mathrm{C}$. The absorbance values were measured using the Multiskan ${ }^{\mathrm{TM}}$ FC Microplate Photometer (Thermo Fisher Scientific, Inc.) at a wavelength of $570 \mathrm{~nm}$.

For the apoptosis assay, $\sim 2.5 \times 10^{6}$ cells cultured in 6-well plates were treated with $0.1 \%$ DMSO or $10 \mu \mathrm{M}$ curcumin. Following $24 \mathrm{~h}$ of treatment, the cells were collected at $500 \mathrm{x} \mathrm{g}$ for $5 \mathrm{~min}$ at room temperature, washed and stained using an Annexin V-Fluorescein Isothiocyanate (FITC)/Propidium Iodide (PI) kit (BD Biosciences, Franklin Lakes, NJ, USA). Briefly, the cells were resuspended in $1 \mathrm{X}$ binding buffer to a concentration of $10^{6}$ cells $/ \mathrm{ml}$. Volumes of $1 \mathrm{ml}$ were transferred to new tubes and $5 \mu 1$ FITC-annexin $\mathrm{V}$ was added prior to incubation for $15 \mathrm{~min}$ at room temperature, and $5 \mu \mathrm{l}$ PI solution was added prior to further incubation for $5 \mathrm{~min}$ at room temperature. The entire process was protected from light. Stained cells were analyzed by the FACSCanto II flow cytometer and BD FACSDiva software (version 6.1.3; BD Biosciences).

For all other assays, NCI-H929 cells were used as they were identified to be more sensitive to curcumin compared with RPMI-8226 cells. Cells were treated with $0.1 \%$ DMSO, $10 \mu \mathrm{M}$ curcumin or $2.5 \mu \mathrm{M}$ 5-aza-2'-deoxycytidine (5-aza-CdR), a demethylation reagent, with $10 \%$ FBS-containing medium for $48 \mathrm{~h}$, and were harvested for DNA, RNA and protein analysis.

Western blotting. Treated cells were harvested and lysed using ice-cold radioimmunoprecipitation assay lysis and extraction buffer (Thermo Fisher Scientific, Inc.) containing a protein inhibitor cocktail (cat. no. P2714; Sigma-Aldrich; Merck KGaA). The protein concentration was determined using a Pierce Rapid Gold Bicinchoninic Acid Protein assay kit (Thermo Fisher Scientific, Inc.) and a NanoDrop 2000 instrument (Thermo Fisher Scientific, Inc.). The proteins $(30 \mu \mathrm{g})$ were separated by SDS-PAGE (12.5\% gel) and transferred to a polyvinylidene difluoride membrane. Subsequently, the membrane was blocked with $5 \%$ fat-free milk in Tris-buffered saline and Tween-20, and sequentially incubated overnight at $4^{\circ} \mathrm{C}$ with 1:500 diluted primary antibodies and $2 \mathrm{~h}$ at room temperature with 1:2,000 diluted horseradish peroxidase-conjugated secondary antibodies (ab6721; Abcam, Cambridge, UK). The signals were detected with the SuperSignal enhanced chemiluminescence detection system (Thermo Fisher Scientific, Inc.) and recorded with the Gel Documentation 2000 system (Bio-Rad Laboratories, Inc., Hercules, CA, USA). Rabbit polyclonal primary antibodies against human methyl CpG-binding protein 2 (MECP2; ab2828), DNMT1 (ab19905), DNMT3a (ab2850), DNMT3b (ab16049) and $\beta$-actin (ab8227) were purchased from Abcam.

DNA extraction and bisulfite genomic sequencing. Genomic DNA was isolated from $\sim 2.5 \times 10^{6}$ treated cells using the QIAamp DNA Mini kit (Qiagen GmbH, Hilden, Germany). Bisulfite-modification of the genomic DNA was performed using the CpGenome ${ }^{\mathrm{TM}}$ Fast DNA Modification kit (Merck $\mathrm{KGaA}$ ). The promoter region of mTOR, which contains a CpG island (chr1:11262153-11263153), was divided into two for bisulfite PCR. Primers for upstream of the first exon of mTOR were as follows: 5'-GTGGTTGTGATAGGTAAA AGATT-3' (forward) and 5'-AACCTAACACAACCCCTC TAAA-3' (reverse); primers for downstream of the first exon of mTOR were as follows: 5'-GAGGGAAGGAGGGTTTTT A-3' (forward) and 5'-CTTTTAATACAATAATTCCTAAAC ACC-3' (reverse). The PCR products were purified and cloned into TOPO vectors (Invitrogen; Thermo Fisher Scientific, Inc.) containing a $\mathrm{T} 7$ promoter near the insert site for sequencing, using a T7 primer (5'-TAATACGACTCACTATAGGG-3'). The overall percentage of the methylation was calculated by dividing the number of methylated $\mathrm{CpGs}$ by the number of total CpGs. The global DNA methylation of treated cells was determined using a Methylamp Global DNA Methylation Quantification kit (EpiGentek, Farmingdale, NY, USA).

$R N A$ isolation and reverse transcription-quantitative PCR (RT-qPCR). Total RNA was extracted from the treated cells using the RNeasy Mini kit (Qiagen $\mathrm{GmbH}$ ). First-strand cDNA was synthesized from $1 \mu \mathrm{g}$ total RNA using the SuperScript III First-Strand Synthesis system for RT-PCR (Invitrogen; Thermo Fisher Scientific, Inc.). The cDNA was used as the template for qPCR (ABI7500; Applied Biosystems; Thermo Fisher Scientific, Inc.). The sequences of the primers used for cDNA amplification were: mTOR 5'-CGCTGTCATCCC 

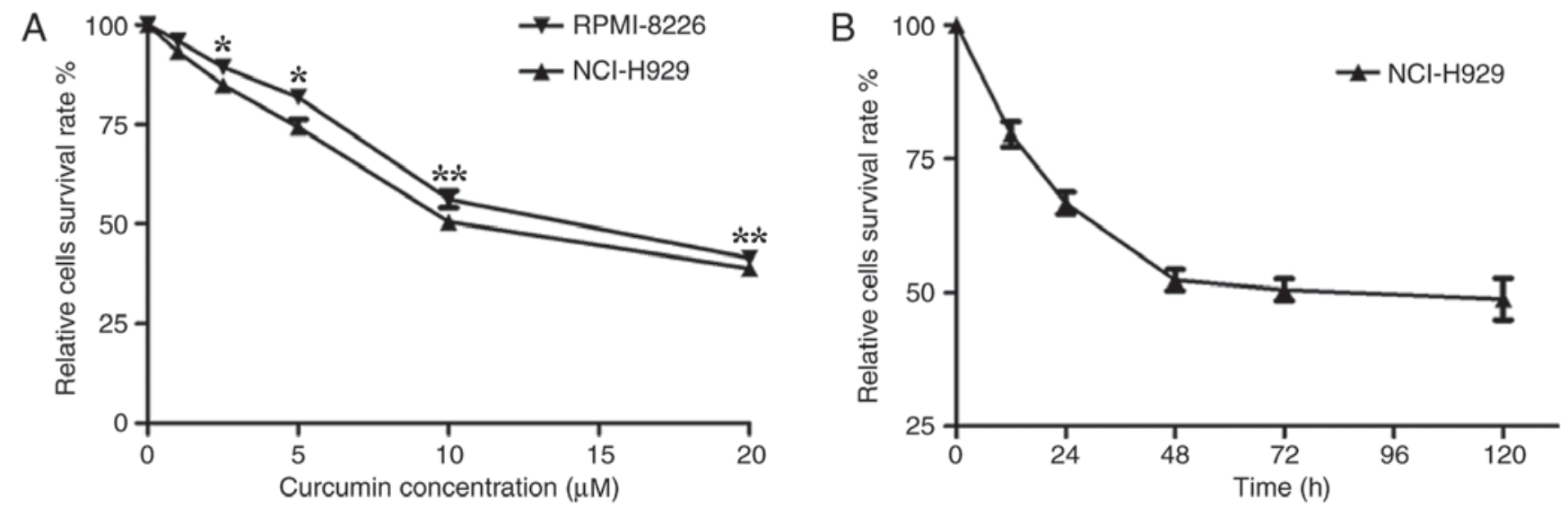

Figure 1. Curcumin suppresses the proliferation of multiple myeloma cells. (A) MTT assays were performed on the multiple myeloma NCI-H929 and RPMI-8226 cell lines to investigate the inhibitory effect of curcumin. (B) Curcumin $(10 \mu \mathrm{M})$ suppressed the proliferation of NCI-H929 cells in a time-dependent manner. ${ }^{*} \mathrm{P}<0.05$, and ${ }^{* * *} \mathrm{P}<0.01$ vs. the untreated group, as calculated by analysis of variance.

TTTATCG-3' (forward) and 5'-ATGCTCAAACACCTCCAC C-3' (reverse), and $\beta$-actin 5'-CTCCATCCTGGCCTCGCT GT-3' (forward) and 5'-GCTGTCACCTTCACCGTTCC-3' (reverse). A Power SYBR ${ }^{\mathrm{TM}}$ Green Master kit (Invitrogen; Thermo Fisher Scientific, Inc.) was used to detect the relative expression of mTOR compared with $\beta$-actin using the $\Delta \Delta \mathrm{Cq}$ method (22). The thermocycling conditions were preheating at $50^{\circ} \mathrm{C}$ for $2 \mathrm{~min}$, denaturation at $95^{\circ} \mathrm{C}$ for $10 \mathrm{~min}$, and 40 amplification cycles of denaturation at $95^{\circ} \mathrm{C}$ for $15 \mathrm{sec}$, and annealing and extension at $60^{\circ} \mathrm{C}$ for $60 \mathrm{sec}$.

Statistical analysis. From the apoptosis, qPCR and western blot assays, the data are presented as the mean \pm standard deviation from 3 independent experiments with duplication of readings. The control and multiple experimental groups were compared using one-way analysis of variance with Dunnett's post hoc analysis. The flow cytometry data between two groups were compared using Student's t-test. All statistics were using the SPSS software, version 17.0 (SPSS Inc., Chicago, IL, USA). $\mathrm{P}<0.05$ was considered to indicate a statistically significant difference.

\section{Results}

Curcumin suppresses the proliferation of multiple myeloma cell lines. It has previously been demonstrated that curcumin influences tumor cell proliferation, apoptosis, migration and invasion, resulting in an antitumor effect (6-11). To examine the effective inhibitory concentration (IC) of curcumin in multiple myeloma cells, an MTT assay was first performed using the human myeloma NCI-H929 and RPMI-8226 cell lines treated with various concentrations of curcumin. The results revealed that curcumin significantly $(\mathrm{P}<0.05)$ suppressed the proliferation of the cells in a dose-dependent manner. The curcumin treatment resulted in a high level of cytotoxicity when the concentration reached $5 \mu \mathrm{M}$ and the highest level was at $20 \mu \mathrm{M}$. The half-maximal IC value of curcumin for these human myeloma cells was $\sim 10 \mu \mathrm{M}$ (Fig. 1A). Compared with the RPMI-8226 cell line, the NCI-H929 cell line was more sensitive to curcumin. Therefore, the subsequent experiments were performed using the NCI-H929 cells, whereby $10 \mu \mathrm{M}$ curcumin was added to the culture medium for different time periods. The MTT assay demonstrated that the number of NCI-H929 cells stabilized when treated with $10 \mu \mathrm{M}$ curcumin for $48 \mathrm{~h}$ or longer (Fig. 1B). These results suggested that the effect of curcumin on NCI-H929 cells was stable after $48 \mathrm{~h}$, determining the time periods chosen for the following experiments.

Curcumin induces apoptosis in multiple myeloma cell lines. To further assess the antitumor effect of curcumin, the multiple myeloma cells were treated with $10 \mu \mathrm{M}$ curcumin for $24 \mathrm{~h}$, and the apoptosis was detected by flow cytometry following Annexin V-FITC/PI staining (Fig. 2A). The apoptosis rates calculated from the RPMI-8226 and NCI-H929 cells were $18.22 \pm 2.50$ and $20.13 \pm 3.55 \%$, respectively, which were significant increases compared with the rates for the corresponding untreated controls $(5.03 \pm 1.46$ and $4.20 \pm 0.95 \%$, respectively) (Fig. 2B).

Curcumin inhibits the expression of mTOR in NCI-H929 cells. In a variety of cancer cells, curcumin can induce apoptosis or autophagy by downregulating the expression of mTOR $(23,24)$. To verify whether curcumin treatment also downregulates the expression of mTOR in the NCI-H929 cell line, the protein expression of mTOR was examined by western blotting and was revealed to be $39.34 \%$ lower with the curcumin treatment than with DMSO treatment (Fig. 3A and B). Furthermore, the RT-qPCR results showed that mTOR mRNA was $43.31 \%$ lower in NCI-H929 cells treated with $10 \mu \mathrm{M}$ curcumin for $48 \mathrm{~h}$ compared with that in the untreated control (Fig. 3C), implying that curcumin inhibits the expression of mTOR by repressing its transcriptional regulation.

Curcumin treatment leads to hypermethylation of the mTOR promoter region. Curcumin has the potential for DNA methylation regulation, and the transcription of associated genes may be directly affected when DNA methylation is altered $(25,26)$. To further determine whether a change in methylation levels was induced by curcumin, the DNA methylation status of the whole genome of NCI-H929 cells treated with $10 \mu \mathrm{M}$ curcumin was determined using the Global DNA Methylation 
A

A

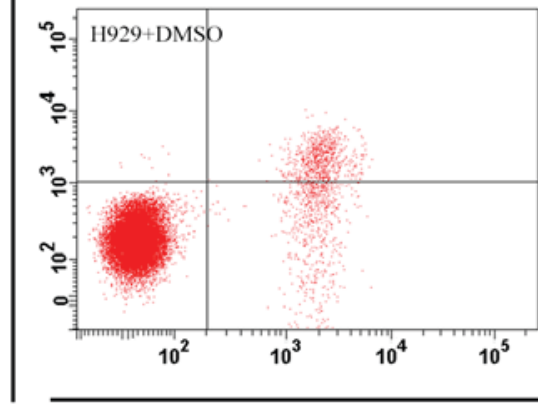

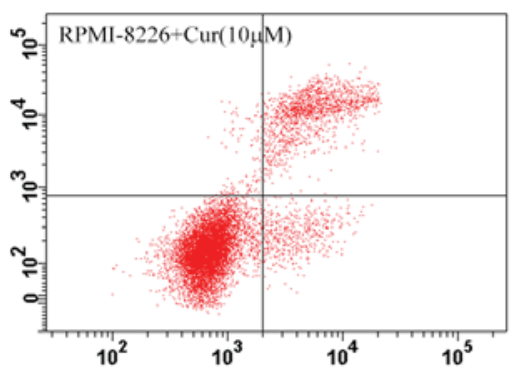

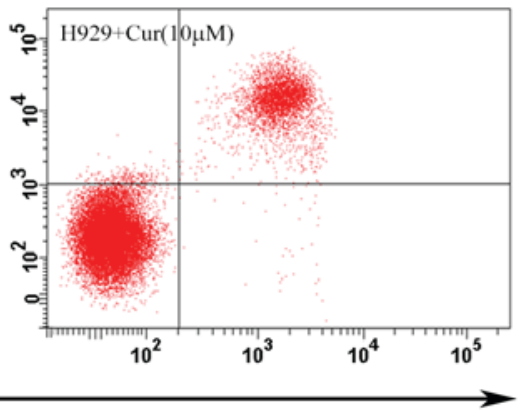

B

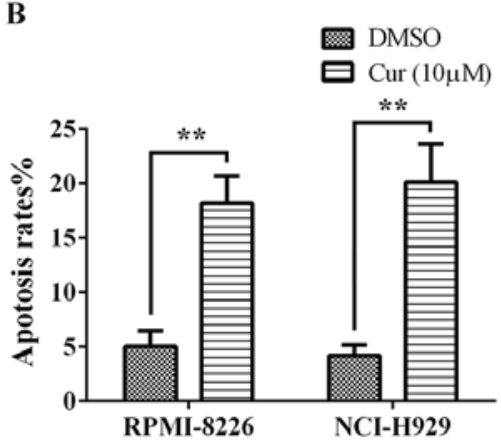

Figure 2. Curcumin induced the apoptosis of multiple myeloma cells. (A) Annexin V-fluorescein isothiocyanate/PI stained RPMI-8226 and NCI-H929 cells treated with $10 \mu \mathrm{M}$ curcumin for $24 \mathrm{~h}$ were detected by flow cytometry. (B) The apoptosis rates of the treated cells as taken from. (A) Each group was analyzed by 3 independent experiments. ${ }^{* *} \mathrm{P}<0.01$ vs. the DMSO group, as calculated by Student's t-test. PI, propidium iodide; Cur, curcumin.
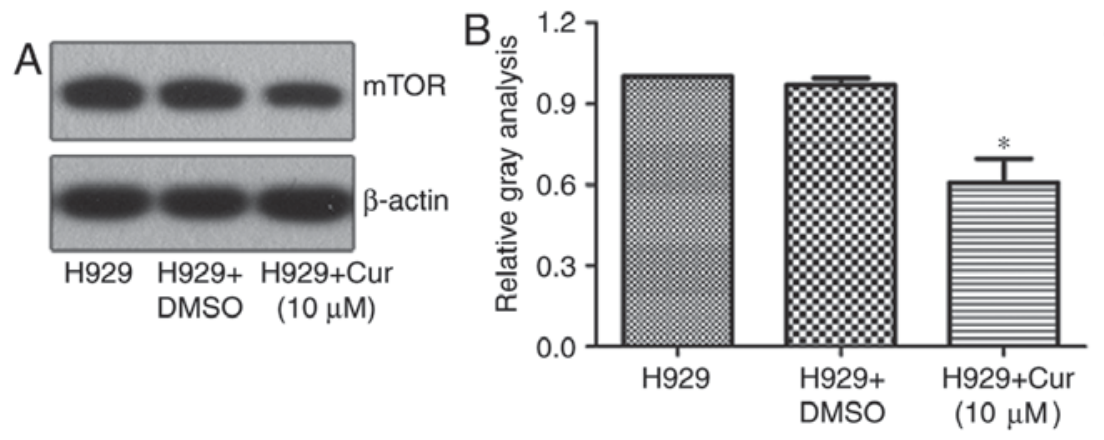

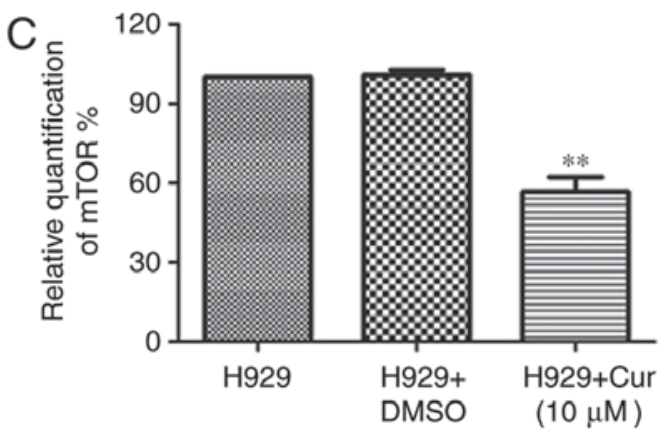

Figure 3. Curcumin inhibits the expression of mTOR in H929 cells. (A) Western blotting determined the levels of mTOR in H929 cells untreated or treated with curcumin or DMSO. (B) Quantitative analysis of the western blotting mTOR protein levels normalized to $\beta$-actin. (C) Relative mRNA expression of mTOR in H929 cells under various treatments $(n=3) .{ }^{*} \mathrm{P}<0.05$ and ${ }^{* *} \mathrm{P}<0.01$, vs. the H929 + DMSO group, as calculated by analysis of variance. H929, NCI-H929; Cur, curcumin; mTOR, mammalian target of rapamycin.

Quantification Ultra kit, and no significant difference was noted compared with the control group (data not shown), indicating that no significant change in DNA methylation on the genomic level occurs following curcumin treatment. However, further bisulfide sequencing revealed that the $\mathrm{CpG}$ island in the mTOR promoter was in a hypermethylated state (Fig. 4).

Curcumin upregulates the expression of DNMT3. Alterations in DNA methylation are mediated by DNMTs. In order to reveal which DNMT participates in the curcumin-induced hypermethylation of the mTOR promoter, the NCI-H929 cells were treated with curcumin and DNA methylation inhibitor 5-aza-CdR for $48 \mathrm{~h}$, and changes in the expression levels of various DNMTs were detected by western blotting (Fig. 5). The levels of the DNMTs were decreased to various degrees in the NCI-H929 cells treated with 5-aza-CdR. In the curcumin-treated cells, the expression of DNMT3a and
DNMT3b, two key methylation transferases of de novo methylation, was significantly upregulated compared with that in the control group. However, there were no significant changes in the expression levels of maintenance methylase DNMT1 and methylation-binding protein MECP2 following treatment with curcumin.

\section{Discussion}

The results of the present study revealed that the expression of mTOR and its promoter methylation in myeloma cells were altered by curcumin, and that this hypermethylation may potentially have been mediated by the upregulation of DNMT3. Curcumin was able to induce apoptosis in 50\% of the myeloma cells when its concentration was increased to $10 \mu \mathrm{M}$. Investigation of the effect of curcumin on normal bone marrow cells was not performed; however, these results 

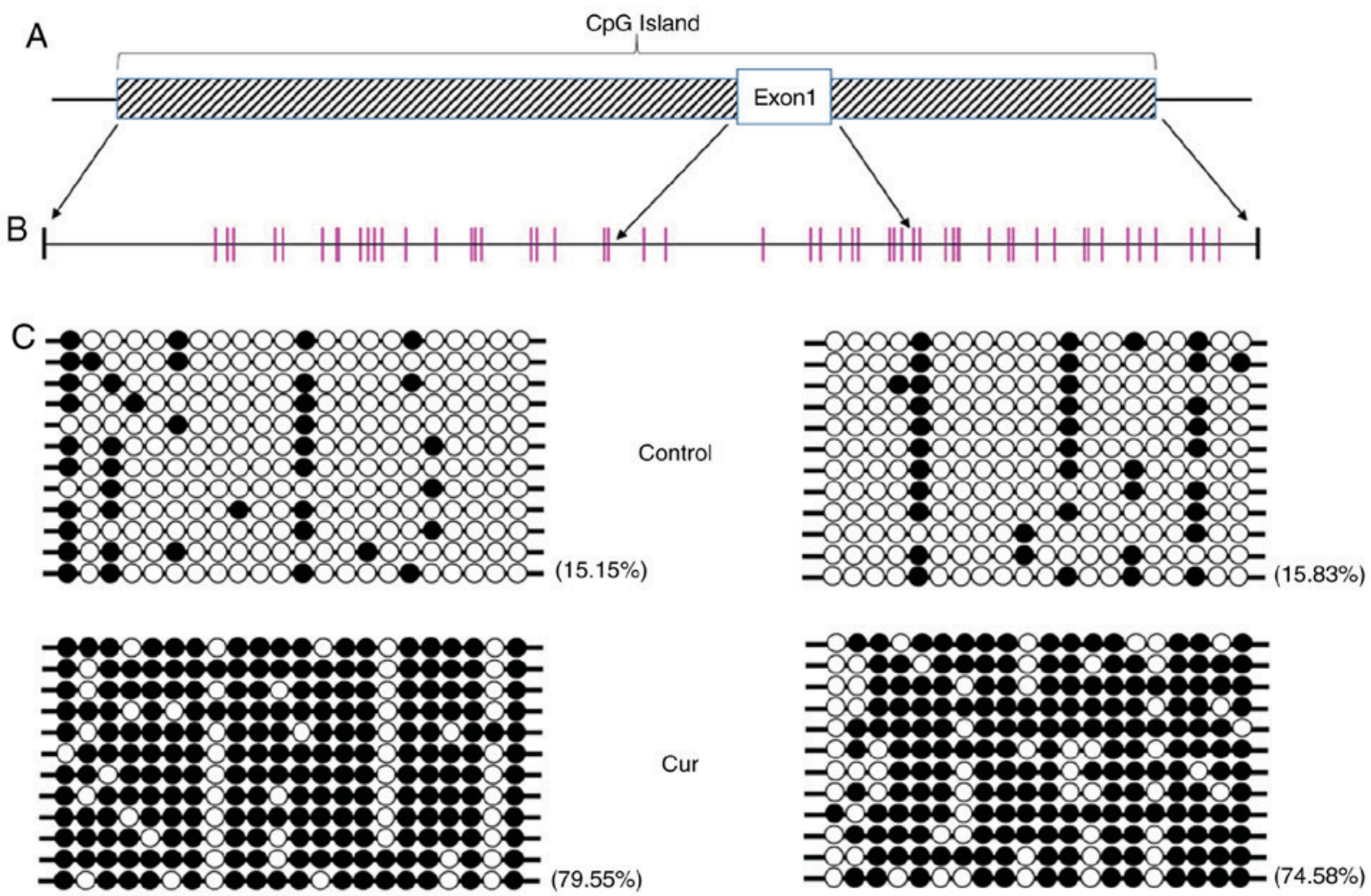

Figure 4. Curcumin hypermethylates the promoter of mTOR in CpG sites. (A) Schematic diagram of the CpG island that begins upstream of the mTOR gene. The striped rectangle represents the $\mathrm{CpG}$ island and the blank rectangle represents mTOR exon 1. (B) CpG sites in the CpG island. Each vertical bar represents a $\mathrm{CpG}$ site. Two regions indicated by four arrows are $\mathrm{CpG}$ islands outside the first exon, which are the target detection areas. (C) The methylation level of the $\mathrm{CpG}$ sites in the promoter of mTOR in the curcumin-treated cells was significantly higher than that that in the control cells. Each line represents a sequencing reaction. The black circles represent methylated $\mathrm{CpG}$ sites, while the white circles represent unmethylated $\mathrm{CpG}$ sites. mTOR, mammalian target of rapamycin; Cur, curcumin.

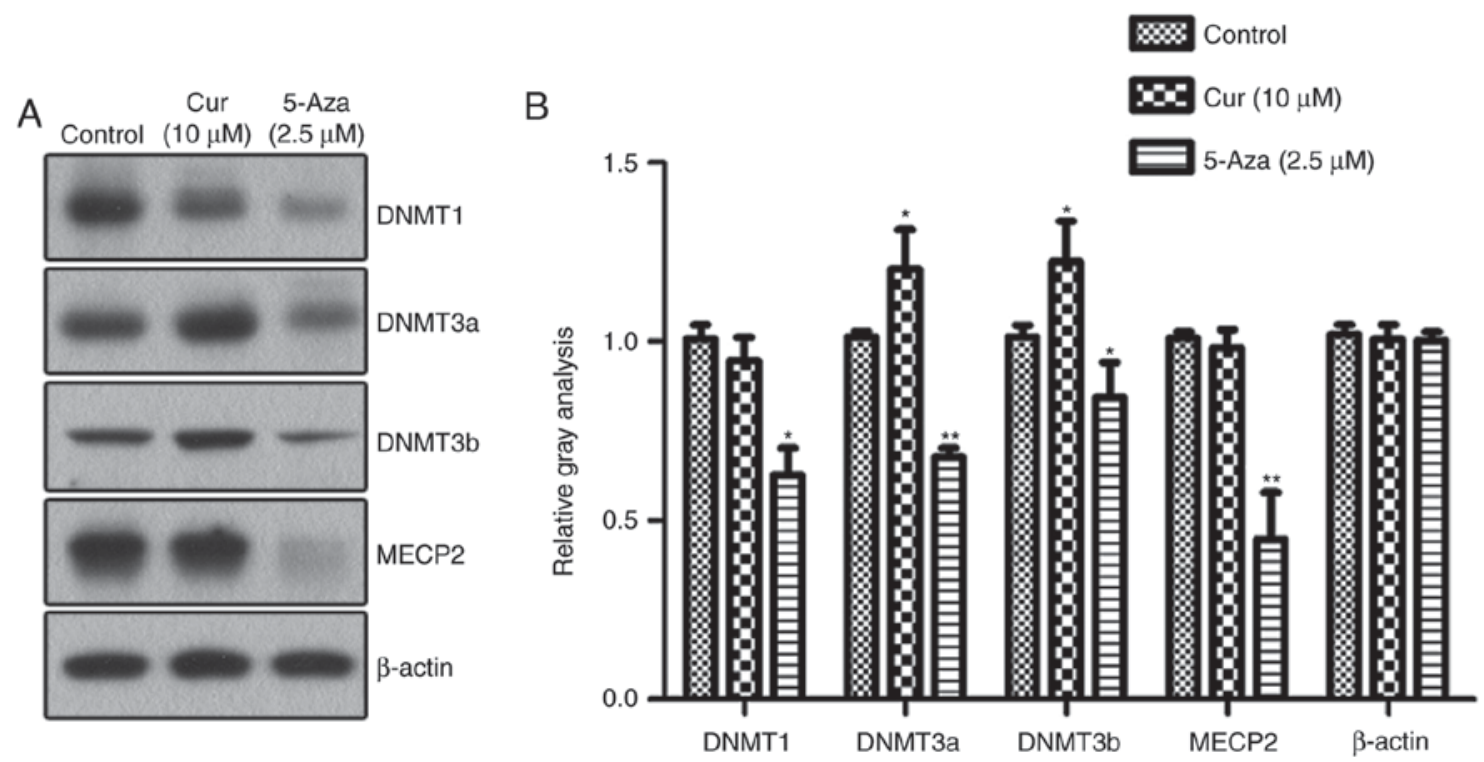

Figure 5. Expression of DNMTs induced by curcumin. (A) Western blotting detected the expression levels of DNMT1, DNMT3a, DNMT3b and MECP2 in NCI-H929 cells following various treatments. (B) Quantitative analysis of the protein levels of DNMT1, DNMT3a, DNMT3b and MECP2, normalized to $\beta$-actin ( $\mathrm{n}=3$ ). " $\mathrm{P}<0.05$ and ${ }^{* * *} \mathrm{P}<0.01$, vs. the control group, as calculated using analysis of variance. DNMT, DNA methyltransferase; MECP2, methyl CpG binding protein 2; Cur, curcumin; 5-Aza, 5-aza-2'-deoxycytidine.

suggest that curcumin may be used in anti-multiple myeloma treatment. Notably, the results indicated that there were no widespread changes in genomic DNA methylation induced by curcumin in NCI-H929 cells, in accordance with the results from colorectal cells in a study by Link et al (25). The present study focused on mTOR, a key factor that activates apoptosis 
and autophagy pathways, rather than performing macroscopic genetic clustering analysis. Lower expression of mTOR and higher promoter methylation were observed, which may be due to changes in DNMT3 expression.

Curcumin is a plant polyphenol extracted from the roots of a plant from the Curcuma genus, and has numerous pharmacological effects, including antitumor, anti-inflammatory, antioxidant and antibacterial properties $(27,28)$. Curcumin may affect cell transcription and regulate apoptosis and autophagy by modulating multiple cell signals, including the nuclear factor- $\kappa \mathrm{B}$, phosphatidylinositol-3-kinase/AKT pathway, the Janus tyrosine kinase/signal transducer and activator of transcription (STAT) signaling transduction pathway and STAT3 $(23,29,30)$. A previous experimental and epidemiological study have proposed that curcumin may alter the DNA methylation status of tumor cells (25); however, its ability to regulate DNA methylase in myeloma cells remains unknown. The present study systematically examined the effect of curcumin on DNMTs in multiple myeloma NCI-H929 cells. To detect the epigenetic regulatory effect of curcumin, 5-aza-CdR was used as a positive control for comparison. Curcumin did not inhibit the expression of methyl-DNA binding protein MECP2 and the maintenance methylase DNMT1 in the NCI-H929 cells, in accordance with the results of the study by Shu et al (31) in LNcaP cells, suggesting that curcumin has no effect on the maintenance of methylation. It has been reported that curcumin is an inhibitor of DNMT1 and may cause a decrease in the overall DNA methylation level in the MV4-11 lymphoma cell line (21). However, in the present study, curcumin was not observed to have an effect on the DNMT1 expression in NCI-H929 cells, but rather it led to an increase in the expression of DNMT3a and DNMT3b. These differences may be due to several factors, including the types of cell lines, the curcumin concentration and the duration of treatment.

In summary, the present study demonstrated that the downregulation of mTOR was associated with hypermethylation of its promoter following treatment with curcumin, which may occur through regulating the expression of DNMT3. It may be concluded that curcumin possesses anti-multiple myeloma activity, which is different from that of chemotherapeutic drugs, including 5-aza-CdR, that cause changes in the overall level of genomic DNA methylation. The precise sites of DNMT3a and DNMT3b that regulate the mTOR promoter and affect its expression should be identified and verified in future studies.

\section{Acknowledgements}

The authors would like to thank Mrs. Baoshan Huang (Wenzhou Medical University, Wenzhou, Zhejiang, China) for assisting with the western blot analysis, and Mr. Lingyun Li, Mr. Zhenqiang Huang and Mr. Youfa Ding (Clinical Laboratory, Lishui People's Hospital, Lishui, Zhejiang, China) for their assistance with the experiments.

\section{Funding}

The present study was supported by Public Projects of Lishui (grant nos. 2014JYZB03 and 2014JYZB16) provided by the Lishui Technology Bureau.

\section{Availability of data and materials}

The datasets used and/or analyzed during the current study are available from the corresponding author on reasonable request.

\section{Authors' contributions}

BF designed the research. JC, YY, HZ, JJ and CQ performed the experiments. JC, TZ, YY and CQ analyzed the data. BF and JC contributed reagents/materials. JC was a major contributor in writing the manuscript. All authors read and approved the final manuscript.

\section{Ethics approval and consent to participate}

Not applicable.

\section{Patient consent for publication}

Not applicable.

\section{Competing interests}

The authors declare that they have no competing interests.

\section{References}

1. Dimopoulos MA and Terpos E: Multiple myeloma. Ann Oncol 21 (Suppl 7): vii143-vii150, 2010.

2. Quach H, Prince HM and Spencer A: Managing multiple myeloma in the elderly: Are we making progress? Expert Rev Hematol 4: 301-315, 2011.

3. Mateos MV, Masszi T, Grzasko N, Hansson M, Sandhu I, Pour L, Viterbo L, Jackson SR, Stoppa AM, Gimsing P, et al: Impact of prior therapy on the efficacy and safety of oral ixazomib-lenalidomide-dexamethasone vs. placebo-lenalidomide-dexamethasone in patients with relapsed/refractory multiple myeloma in TOURMALINE-MM1. Haematologica 102: 1767-1775, 2017.

4. Moreau P, Masszi T, Grzasko N, Bahlis NJ, Hansson M, Pour L, Sandhu I, Ganly P, Baker BW, Jackson SR, et al: Oral Ixazomib, Lenalidomide, and Dexamethasone for Multiple Myeloma. N Engl J Med 374: 1621-1634, 2016.

5. Howells LM, Mitra A and Manson MM: Comparison of oxaliplatin- and curcumin-mediated antiproliferative effects in colorectal cell lines. Int J Cancer 121: 175-183, 2007.

6. Jin G, Yang Y, Liu K, Zhao J, Chen X, Liu H, Bai R, Li X, Jiang Y, Zhang X, et al: Combination curcumin and (-)-epigallocatechin-3-gallate inhibits colorectal carcinoma microenvironment-induced angiogenesis by JAK/STAT3/IL-8 pathway. Oncogenesis 6: e384, 2017.

7. Weissenberger J, Priester M, Bernreuther C, Rakel S, Glatzel M, Seifert V and Kögel D: Dietary curcumin attenuates glioma growth in a syngeneic mouse model by inhibition of the JAK1,2/STAT3 signaling pathway. Clin Cancer Res 16: 5781-5795, 2010.

8. Zanotto-Filho A, Braganhol E, Klafke K, Figueiró F, Terra SR, Paludo FJ, Morrone M, Bristot IJ, Battastini AM, Forcelini CM, et al: Autophagy inhibition improves the efficacy of curcumin/temozolomide combination therapy in glioblastomas. Cancer Lett 358: 220-231, 2015.

9. Ye M, Zhang J, Zhang J, Miao Q, Yao L and Zhang J: Curcumin promotes apoptosis by activating the p53-miR-192-5p/215-XIAP pathway in non-small cell lung cancer. Cancer Lett 357: 196-205, 2015.

10. Srivastava RK, Chen Q, Siddiqui I, Sarva K and Shankar S: Linkage of curcumin-induced cell cycle arrest and apoptosis by cyclin-dependent kinase inhibitor p21(/WAF1/CIP1). Cell Cycle 6: 2953-2961, 2007.

11. Shinojima N, Yokoyama T, Kondo Y and Kondo S: Roles of the Akt/mTOR/p70S6K and ERK1/2 signaling pathways in curcumin-induced autophagy. Autophagy 3: 635-637, 2007. 
12. Cao P, Maira SM, García-Echeverría C and Hedley DW: Activity of a novel, dual PI3-kinase/mTor inhibitor NVP-BEZ235 against primary human pancreatic cancers grown as orthotopic xenografts. Br J Cancer 100: 1267-1276, 2009.

13. Nassim R, Mansure JJ, Chevalier S, Cury F and Kassouf W: Combining mTOR inhibition with radiation improves antitumor activity in bladder cancer cells in vitro and in vivo: A novel strategy for treatment. PLoS One 8: e65257, 2013.

14. Tasian SK, Teachey DT, Li Y, Shen F, Harvey RC, Chen IM, Ryan T, Vincent TL, Willman CL, Perl AE, et al: Potent efficacy of combined PI3K/mTOR and JAK or ABL inhibition in murine xenograft models of $\mathrm{Ph}$-like acute lymphoblastic leukemia. Blood 129: 177-187, 2017.

15. Zoncu R, Efeyan A and Sabatini DM: mTOR: From growth signal integration to cancer, diabetes and ageing. Nat Rev Mol Cell Biol 12: 21-35, 2011.

16. Suangtamai T and Tanyong DI: Diallyl disulfide induces apoptosis and autophagy via mTOR pathway in myeloid leukemic cell line. Tumour Biol 37: 10993-10999, 2016.

17. Wang FM, Galson DL, Roodman GD and Ouyang H: Resveratrol triggers the pro-apoptotic endoplasmic reticulum stress response and represses pro-survival XBP1 signaling in human multiple myeloma cells. Exp Hematol 39: 999-1006, 2011.

18. Balasubramanyam K, Varier RA, Altaf M, Swaminathan V, Siddappa NB, Ranga U and Kundu TK: Curcumin, a novel p300/CREB-binding protein-specific inhibitor of acetyltransferase, represses the acetylation of histone/nonhistone proteins and histone acetyltransferase-dependent chromatin transcription. J Biol Chem 279: 51163-51171, 2004.

19. Kang J, Chen J, Shi Y, Jia J and Zhang Y: Curcumin-induced histone hypoacetylation: The role of reactive oxygen species. Biochem Pharmacol 69: 1205-1213, 2005.

20. Kang SK, Cha SH and Jeon HG: Curcumin-induced histone hypoacetylation enhances caspase-3-dependent glioma cell death and neurogenesis of neural progenitor cells. Stem Cells Dev 15: 165-174, 2006.

21. Liu Z, Xie Z, Jones W, Pavlovicz RE, Liu S, Yu J, Li PK, Lin J, Fuchs JR, Marcucci G, et al: Curcumin is a potent DNA hypomethylation agent. Bioorg Med Chem Lett 19: 706-709, 2009.

22. Livak KJ and Schmittgen TD: Analysis of relative gene expression data using real-time quantitative PCR and the 2(-Delta Delta C(T)) method. Methods 25: 402-408, 2001.
23. Guo Y, Shan Q, Gong Y, Lin J, Shi F, Shi R and Yang X: Curcumin induces apoptosis via simultaneously targeting AKT/mTOR and $\mathrm{RAF} / \mathrm{MEK} / \mathrm{ERK}$ survival signaling pathways in human leukemia THP-1 cells. Pharmazie 69: 229-233, 2014.

24. Zhao G, Han X, Zheng S, Li Z, Sha Y, Ni J, Sun Z, Qiao S and Song Z: Curcumin induces autophagy, inhibits proliferation and invasion by downregulating AKT/mTOR signaling pathway in human melanoma cells. Oncol Rep 35: 1065-1074, 2016.

25. Link A, Balaguer F, Shen Y, Lozano JJ, Leung HC, Boland CR and Goel A: Curcumin modulates DNA methylation in colorectal cancer cells. PLoS One 8: e57709, 2013.

26. Wu P, Huang R, Xiong YL and Wu C: Protective effects of curcumin against liver fibrosis through modulating DNA methylation. Chin J Nat Med 14: 255-264, 2016.

27. Agrawal DK and Mishra PK: Curcumin and its analogues: Potential anticancer agents. Med Res Rev 30: 818-860, 2010.

28. Naksuriya O, Okonogi S, Schiffelers RM and Hennink WE: Curcumin nanoformulations: A review of pharmaceutical properties and preclinical studies and clinical data related to cancer treatment. Biomaterials 35: 3365-3383, 2014.

29. Marquardt JU, Gomez-Quiroz L, Arreguin Camacho LO, Pinna F, Lee YH, Kitade M, Dominguez MP, Castven D, Breuhahn K, Conner EA, et al: Curcumin effectively inhibits oncogenic NF- $\mathrm{KB}$ signaling and restrains stemness features in liver cancer. J Hepatol 63: 661-669, 2015.

30. Hu A, Huang JJ, Jin XJ, Li JP, Tang YJ, Huang XF, Cui HJ, $\mathrm{Xu} \mathrm{WH}$ and Sun GB: Curcumin suppresses invasiveness and vasculogenic mimicry of squamous cell carcinoma of the larynx through the inhibition of JAK-2/STAT-3 signaling pathway. Am J Cancer Res 5: 278-288, 2014.

31. Shu L, Khor TO, Lee JH, Boyanapalli SS, Huang Y, Wu TY, Saw CL, Cheung KL and Kong AN: Epigenetic CpG demethylation of the promoter and reactivation of the expression of Neurog1 by curcumin in prostate LNCaP cells. AAPS J 13: 606-614, 2011.

(i) $($ ) This work is licensed under a Creative Commons

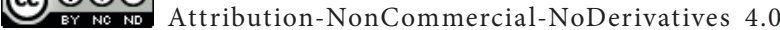
International (CC BY-NC-ND 4.0) License. 